Preprint typeset in JHEP style - HYPER VERSION

hep-ph/0310095

\title{
Chiral Symmetry in Light-front QCD
}

\author{
Meng-Hsiu Wu \\ Department of Physics, National Cheng Kung University, Tainan, 701, Taiwan \\ E-mail: mhwu@hepth.phys.ncku.edu.tw \\ Wei-Min Zhang \\ Department of Physics, National Cheng Kung University, Tainan, 701, Taiwan \\ and National Center for Theoretical Science, Taiwan \\ E-mail: wzhang@mail.ncku.edu.tw
}

\begin{abstract}
The definition of chiral transformations in light-front field theory is very different from the conventional form in equal-time formalism. We study the consistency of chiral transformations and chiral symmetry in light-front QCD and derive a complete new light-front axial-vector current for QCD. The breaking of chiral symmetry in light-front QCD is only associated with helicity flip interaction between quarks and gluons. Remarkably, the new axial-vector current does not contain the pion pole part so that the associate chiral charge smoothly describes pion transitions for various hadronic processes.
\end{abstract}

KEYWORDs: Global Symmetries, QCD. 


\section{Introduction}

In the investigations of the nonperturbative QCD in light-front theory, the most difficult problem is how to provide a clear picture of the dynamical chiral symmetry breaking and the quark confinement with a trivial light-front QCD vacuum [1]. The trivial vacuum in light-front theory is obtained by removing the longitudinal zero modes which can be easily realized by imposing an explicit cutoff on small longitudinal momentum [1] or via a suitable prescription on the operator $1 / \partial^{+}$[2]. Both methods give a specific regularization of the longitudinal momentum going to be zero. It is then natural to ask how the spontaneous chiral symmetry breaking (which is a nontrivial vacuum effect of QCD in common understanding and plays a crucial role in the description of low energy hadronic physics) can be manifested in light-front formulation. A few years ago, Wilson pointed out that the concept of spontaneous chiral symmetry breaking may be realized via an explicit symmetry breaking in light-front theory induced from counterterms of removing longitudinal infrared momentum divergence [3], but up to date a practical scheme of this procedure has not been carried out yet.

In fact, chiral symmetry itself is not a good dynamical symmetry for strong interaction. Mechanism of dynamical symmetry breaking can manifest differently in different space-time frameworks 四, 5]. Therefore, before we attempt to derive the counterterms related to chiral symmetry breaking through a reliable renormalization procedure for the light-front QCD Hamiltonian, it is certainly helpful to understand precisely the concept of chiral symmetry and its breaking in light-front theory. In this paper, we shall study chiral transformations in light-front theory and the corresponding divergence of axial-vector current for light-front QCD. We will then further explore its relation with pionic dynamics and the associated physical implication in hadronic structure.

If we do not take into account the anomaly, the axial-vector current, defined by $j_{5}^{\mu} \equiv$ $\bar{\psi} \gamma^{\mu} \gamma_{5} \psi$, satisfies the following equation:

$$
\partial_{\mu} j_{5}^{\mu}=2 i m \bar{\psi} \gamma_{5} \psi
$$

namely, the axial-vector current is not conserved for massive fermion theory no matter the fundamental theory is a free theory or an interacting theory. The divergence of axial-vector current is only related to fermion mass and is independent on the detailed interacting structure of the underlying particles. However, in light-front theory, a fermion field is separated into an up and a down components, $\psi=\psi_{+}+\psi_{-}$, and only the up component field $\left(\psi_{+}\right)$is a dynamical variable. Thus, chiral dynamics of a fermion in light-front theory might behave differently from that in equal-time formulation. In fact, it was noted long time ago [6] that since the fermion field $\psi$ mix the left- and right-handed components in light-front theory, the usual chiral transformation can only be applied to the up component $\psi_{+}$. The transformation for down component $\psi_{-}$is determined by the light-front fermion constraint. As a result, it can be shown that the standard axial-vector current $j_{5}^{\mu}$ is not a Noether current associated with such a light-front chiral transformation [7]. The new light-front axial-vector current with respect to the light-front chiral transformation obeys a divergence equation that differs from eq. (1.1). In recent years, similar idea has been 
applied to chiral Yukawa model, Nambu-Jona-Lasinio model [8] and light-front $\mathrm{O}(2)$ sigma model with fermions [9], but up to date none has explored seriously about chiral dynamics for light-front QCD from this point of view. Using such a consistent light-front chiral transformation, we find that the conservation law of the axial-vector current in light-front theory is broken only by the helicity flip interaction alone in the QCD Lagrangian and the new light-front axial-vector current does not contain the pion-pole contribution. This may provide us a new look about the chiral symmetry and chiral dynamics of QCD in light-front theory.

The paper is organized as follows: In Section 2, we explore in details chiral transformations in light-front theory and chiral symmetry in light-front QCD. In Section 3, we use the point-splitting technique to derive the chiral anomalies. In Section \& we discuss the physical implication of the new light-front axial-vector current. A conclusion is given in section 5 .

\section{Chiral transformation in the light-front QCD}

Before we discuss chiral symmetry in light-front QCD, we consider first a free fermion theory for an illustration. Explicitly, the free fermion Lagrangian in the light-front coordinates with $x^{+}=x^{0}+x^{3}$ as the "time" direction can be written as

$$
\mathcal{L}=\bar{\psi}(i \not \partial-m) \psi=-\psi_{+}^{\dagger} \frac{\square^{2}+m^{2}}{i \partial^{+}} \psi_{+},
$$

where $\psi_{+}$is called the up component of fermion field: $\psi=\psi_{+}+\psi_{-}$with the definition $\psi_{ \pm} \equiv \frac{1}{4} \gamma^{\mp} \gamma^{ \pm} \psi \equiv \Lambda_{ \pm} \psi\left(\gamma^{ \pm}=\gamma^{0} \pm \gamma^{3}\right)$, and the down component is determined by the light-front fermion constraint

$$
\psi_{-}=\frac{1}{i \partial^{+}}\left(i \vec{\alpha}_{\perp} \cdot \vec{\partial}_{\perp}+\beta m\right) \psi_{+},
$$

while $\square^{2}$ is the Laplace operator: $\square^{2}=\partial^{+} \partial^{-}-\partial_{\perp}^{2}$, and the operator $\frac{1}{\partial^{+}}$is defined to satisfy the identity $\left(\frac{1}{\partial^{+}}\right) \partial^{+}=\partial^{+} \frac{1}{\partial^{+}}=1$. An explicit regularization of this operator is given by [2]

$$
\left(\frac{1}{\partial^{+}}\right) f\left(x^{+}, x^{-}, \vec{x}_{\perp}\right)=\frac{1}{4} \int_{-\infty}^{\infty} \mathrm{d} x^{\prime-} \epsilon\left(x^{-}-x^{\prime-}\right) f\left(x^{+}, x^{\prime-}, \vec{x}_{\perp}\right),
$$

and $\epsilon(x)=1,0,-1$ for $x>0,=0,<0$.

Eq. (2.1) is obvious chiral invariant regardless of that the fermion is massive or massless. This is because the light-front Lagrangian in eq. (2.1) does not contain explicitly gamma matrix and therefore is invariant under chiral transformations. This contradicts with eq. (1.1) that the axial-vector current is not conserved. Moreover, the chiral charge in light-front $Q_{5}^{L}$ is defined by

$$
Q_{5}^{L} \equiv \frac{1}{2} \int \mathrm{d} x^{-} \mathrm{d}^{2} x_{\perp} j_{5}^{+}=\int \mathrm{d} x^{-} \mathrm{d}^{2} x_{\perp} \psi_{+}^{\dagger} \gamma_{5} \psi_{+}
$$


which commutes with the free-fermion light-front Hamiltonian

$$
\partial^{-} Q_{5}^{L}=\left[Q_{5}^{L}, H_{L F}\right]=0
$$

where

$$
H_{L F}=\int \mathrm{d} x^{-} \mathrm{d}^{2} x_{\perp}\left(i \psi_{+}^{\dagger} \partial^{-} \psi_{+}-\mathcal{L}\right)=\int \mathrm{d} x^{-} \mathrm{d}^{2} x_{\perp}\left(\psi_{+}^{\dagger} \frac{-\nabla_{\perp}^{2}+m^{2}}{i \partial^{+}} \psi_{+}\right)
$$

In other words, the light-front Hamiltonian of a free massive fermion theory is chiral symmetric. Thus, chiral symmetry in light-front field theory is apparently inconsistent that the free massive light-front Lagrangian is chiral invariant and the chiral charge is conserved but the axial-vector current $j_{5}^{\mu}$ is not [see eq. (1.1)].

In ref. [1], it has been argued that this inconsistency of the conserved light-front chiral charge with the nonconserved axial-vector current occurs due to an improper treatment of the zero longitudinal momentum (i.e., so-called zero modes) in light-front theory. From the current divergence equation, the light-front time derivative of the chiral charge is proportional to $\int \mathrm{d} x^{-} \mathrm{d}^{2} x_{\perp} \bar{\psi} \gamma_{5} \psi$. When the fields in this integral are expanded in terms of momentum eigenstates, the diagonal terms $b^{\dagger} b$ and $d^{\dagger} d$, where $b$ and $d$ are the quark and antiquark annihilation operators, vanish; namely, the matrix elements multiplying them vanish. Moreover, the off-diagonal terms vanish if the zero modes are absent. Thus, it seems to be the absence of zero modes that makes it possible for the light-front chiral charge to be conserved irrespective of the nonconservation of the axial-vector current for free massive fermions [1]. Since the longitudinal momentum of any particle cannot be negative in light-front theory, the light-front vacuum is only occupied by particles with zero longitudinal momentum. Undoubtedly, light-front longitudinal zero modes play an important role in understanding nontrivial vacuum structures of field theory. However, the above inconsistency occurs in a free theory. The vacuum of a free theory is trivial no matter whether there exist zero modes or not, and the explicit chiral symmetry breaking of a free massive fermionic theory must be irrelevant to zero modes.

In fact, the apparent inconsistency between eq. (1.1) and eq. 2.5) originates from the improper definition of the axial-vector current from the chiral transformation in lightfront theory [7]. According to Noether's theorem, a current is defined from the associated continuous transformation. In light-front theory, as we have seen the fermion field $\psi$ is divided into $\psi_{+}$and $\psi_{-}$, only one of them is a dynamical independent variable. It is easy to check that the usual chiral transformation,

$$
\psi(x) \mapsto \psi^{\prime}(x)=\mathrm{e}^{-i \theta \gamma_{5}} \psi(x)
$$

is inconsistent with eq. (2.2). The light-front fermion constraint eq. (2.2) is derived from the fermion equation of motion and therefore it must be obeyed. Thus, the chiral transformation can only be applied to $\psi_{+}$,

$$
\psi_{+}(x) \mapsto \psi_{+}^{\prime}(x)=\mathrm{e}^{-i \theta \gamma_{5}} \psi_{+}(x)
$$


and the chiral transformation for $\psi_{-}$is determined by eq. (2.2),

$$
\begin{aligned}
\psi_{-}(x) \mapsto \psi_{-}^{\prime}(x) & =\frac{1}{i \partial^{+}}\left(i \vec{\alpha}_{\perp} \cdot \vec{\partial}_{\perp}+\beta m\right) \psi_{+}^{\prime}(x) \\
& =\mathrm{e}^{-i \theta \gamma_{5}} \psi_{-}+m \gamma^{0}\left(\mathrm{e}^{-i \theta \gamma_{5}}-\mathrm{e}^{i \theta \gamma_{5}}\right) \frac{1}{i \partial^{+}} \psi_{+}(x) .
\end{aligned}
$$

To obtain a consistent light-front axial-vector current from the above chiral transformation, we start from the light-front Lagrangian eq. (2.1) for an infinitesimal transformation i.e., $\psi_{+}^{\prime}=\left(1-i \theta \gamma_{5}\right) \psi_{+}$. The result is

$$
\mathcal{L}^{\prime}(x)=\mathcal{L}(x)-\theta \partial_{\mu} \tilde{j}_{5}^{\mu}
$$

where $\tilde{j}_{5}^{\mu}$ is given by

$$
\begin{aligned}
\tilde{j}_{5}^{\mu} & =\bar{\psi} \gamma^{\mu} \gamma_{5} \psi-m \bar{\psi} \gamma^{\mu} \gamma_{5} \gamma^{+} \frac{1}{i \partial^{+}} \psi \\
& =j_{5}^{\mu}-\hat{j}_{5}^{\mu} .
\end{aligned}
$$

This is the new axial-vector current with respect to the light-front chiral transformation eq. (2.8). Eq. (2.10) shows that under the light-front chiral transformation, the free fermion Lagrangian is invariant up to a total derivative term. By the Noether's theorem, we have

$$
\partial_{\mu} \tilde{j}_{5}^{\mu}=0
$$

In other word, for a free fermion theory in light-front, the chiral symmetry is conserved and the conserved axial-vector current is given by $\tilde{j}_{5}^{\mu}$ of eq. (2.11).

Furthermore, it is remarkable to see that, although the light-front axial-vector current $\tilde{j}_{5}^{\mu}$ is different from the conventional one $j_{5}^{\mu}$, the $\tilde{j}_{5}^{+}$component is the same as $j_{5}^{+}$component, and so does the light-front chiral charge,

$$
\tilde{Q}_{5}^{L} \equiv \frac{1}{2} \int \mathrm{d} x^{-} \mathrm{d}^{2} x_{\perp} \tilde{j}_{5}^{+}=\int \mathrm{d} x^{-} \mathrm{d}^{2} x_{\perp} j_{5}^{+}=Q_{5}^{L}
$$

This is why even if we define the light-front chiral charge $Q_{5}^{L}$ from the conventional definition in the beginning, it is still a conserved one. The conservation of chiral charge in light-front theory is just a consequence of eq. (2.12). Physically, this result is easy to be understood. A free light-front fermion theory is chiral invariant since the light-front chiral charge operator is identical to the fermion's helicity operator, no matter the fermion is massive or massless. Explicitly, the chirality of a fermion is characterized by the chiral charge in light-front theory, $\tilde{Q}_{5}^{L}$ defined by eq. (2.13). In the momentum space,

$$
\tilde{Q}_{5}^{L}=\int \frac{\mathrm{d} p^{+} \mathrm{d}^{2} \vec{p}_{\perp}}{2(2 \pi)^{3}} \sum_{\lambda= \pm 1 / 2} 2 \lambda\left[b^{\dagger}(p, \lambda) b(p, \lambda)+d^{\dagger}(p,-\lambda) d(p,-\lambda)\right],
$$

where $\lambda$ denotes the helicity. Thus, the chirality is identical to the helicity in light-front theory. If the Lagrangian does not contain helicity flip interactions, the helicity as well as the chirality are always conserved. 
Moreover, from eqs. (2.11) and (2.12), one can easily reproduce eq. (11.1) i.e.,

$$
\partial_{\mu} j_{5}^{\mu}=2 i m \bar{\psi} \gamma_{5} \psi
$$

Thus we have reached to a consistent solution that a free fermion theory in light-front, no matter if it is a massless or massive theory, is chiral invariant but the light-front axialvector current must be defined in terms of eq. (2.11). An alternative discussion of the light-front axial-vector current based on the definition of Noether current for a free theory has also been given by Mustaki []. In recent years, one has also use such new light-front axial-vector to study chiral dynamics in chiral Yukawa model and Nambu-Jona-Lasinio model [8, 9]. However, up to date, none has explored carefully about the chiral symmetry for light-front QCD from this point of view.

Now we consider QCD. We start from light-front QCD Lagrangian (namely, the QCD Lagrangian expressed in terms of all the physical light-front field variables [2])

$$
\mathcal{L}_{\mathrm{LFQCD}}=\bar{\psi}(i \not D-m) \psi-\frac{1}{2} \operatorname{Tr}\left(F_{\mu \nu} F^{\mu \nu}\right)
$$

where we shall take the light-front gauge $A_{a}^{+}=0$, then $\psi=\psi_{+}+\psi_{-}$such that $\psi_{-}$is determined from the QCD Dirac equation,

$$
\psi_{-}=\frac{1}{i \partial^{+}}\left(i \vec{\alpha}_{\perp} \cdot \vec{\partial}_{\perp}+g \vec{\alpha}_{\perp} \cdot \vec{A}_{\perp}+\beta m\right) \psi_{+} .
$$

In light-front QCD, the chiral transformation of $\psi_{+}$is defined as the same as eq. (2.8) but the corresponding transformation of $\psi_{-}$is determined from eq. (2.16). The light-front axial-vector current can be obtained through an infinitesimal chiral transformation,

$$
\psi_{+}(x) \mapsto \psi_{+}^{\prime}(x)=\left(1-i \gamma_{5} \theta\right) \psi_{+}(x)
$$

to the LFQCD action $\mathcal{S}=\int \mathrm{d}^{4} \tilde{x} \mathcal{L}_{\mathrm{LFQCD}}$. Under these transformations, we find that

$$
\mathcal{L}_{\mathrm{LFQCD}}^{\prime}(x)=\mathcal{L}(x)-\theta\left[\partial_{\mu} \tilde{j}_{5}^{\mu}-2 m g \psi_{+}^{\dagger} \gamma_{5}\left(\frac{1}{\partial^{+}} \vec{\gamma}_{\perp} \cdot \vec{A}_{\perp}\right) \psi_{+}\right]
$$

where the light-front axial-vector current $\tilde{j}_{5}^{\mu}$ for QCD is given the same as eq.(2.11),

$$
\begin{aligned}
\tilde{j}_{5}^{\mu} & =\bar{\psi} \gamma^{\mu} \gamma_{5} \psi-m \bar{\psi} \gamma^{\mu} \gamma_{5} \gamma^{+} \frac{1}{i \partial^{+}} \psi \\
& =j_{5}^{\mu}-\hat{j}_{5}^{\mu}
\end{aligned}
$$

except that now the constraint of $\psi_{-}$is determined by eq. (2.16). Compare with eq. (2.10) and eq. (2.18), one can see that the light-front QCD Lagrangian is not invariant under the light-front chiral transformation eq. (2.17). It is easy to check by equations of motion that the divergence of $\tilde{j}_{5}^{\mu}$ is given by

$$
\begin{aligned}
\partial_{\mu} \tilde{j}_{5}^{\mu} & =-2 m g \psi_{+}^{\dagger} \gamma_{5}\left(\frac{1}{\partial^{+}} \vec{\gamma}_{\perp} \cdot \vec{A}_{\perp}\right) \psi_{+} \\
& =m g \bar{\psi} \gamma_{5} \gamma^{+}\left(\frac{1}{\partial^{+}} \vec{\gamma}_{\perp} \cdot \vec{A}_{\perp}\right) \psi
\end{aligned}
$$


The right-hand side of the above equation is only related to the term in the light-front QCD Lagrangian that flips quark's helicity through its interaction with gluons. It tells us that the manifestation of chiral symmetry breaking in light-front QCD is different from that in equal-time formalism. Here the chiral symmetry of light-front QCD is broken due to quark-gluon interaction involving a helicity flip process. Meanwhile, from the new lightfront axial-vector current, one can check that eq. (2.20) is still consistent with the familiar divergent equation of the conventional axial-vector current eq. (1.1), namely,

$$
\begin{aligned}
\partial_{\mu} \tilde{j}_{5}^{\mu} & =\partial_{\mu} j_{5}^{\mu}-m \partial_{\mu}\left(\bar{\psi} \gamma^{\mu} \gamma_{5} \gamma^{+} \frac{1}{i \partial^{+}} \psi\right) \\
& =m g \bar{\psi} \gamma_{5} \gamma^{+}\left(\frac{1}{\partial^{+}} \vec{\gamma}_{\perp} \cdot \vec{A}_{\perp}\right) \psi
\end{aligned}
$$

which leads to the familiar form in equal-time formalism.

$$
\partial_{\mu} j_{5}^{\mu}=2 i m \bar{\psi} \gamma_{5} \psi
$$

Furthermore, we can see from eq. (2.19) that the light-front axial-vector current is just the conventional axial-vector current subtracting an additional term $\hat{j}_{5}^{\mu}$. As we will discuss in Sec. IV, the operator $\hat{j}_{5}^{\mu}$ is related to the pion pole contribution in hadronic transition processes.

To further check the consistency of the light-front chiral transformation obtained here, we may calculate the commutator of $\psi_{+}$and $\tilde{Q}_{5}^{L}=Q_{5}^{L}$ in light-front theory: using the light-front canonical quantization rule,

$$
\left\{\psi_{+}(x), \psi_{+}^{\dagger}(y)\right\}_{x^{+}=y^{+}}=\Lambda_{+} \delta^{3}(x-y)
$$

it is easy to find that

$$
\left[\psi_{+}(x), Q_{5}^{L}\right]=\gamma_{5} \psi_{+}(x)
$$

This corresponds to the infinitesimal chiral transformation of eq. (2.8). The corresponding commutator of $\psi_{-}$can be evaluated using eqs. (2.16), (2.22) and (2.23), with the result

$$
\left[\psi_{-}(x), Q_{5}^{L}\right]=\gamma_{5} \psi_{-}(x)-m \gamma_{5} \gamma^{+} \frac{1}{i \partial^{+}} \psi_{+}(x)
$$

which gives the infinitesimal chiral transformation of eq. (2.9). Then the transformation of quark field under $Q_{5}^{L}$ is given by

$$
\left[\psi(x), Q_{5}^{L}\right]=\gamma_{5} \psi(x)-m \gamma_{5} \gamma^{+} \frac{1}{i \partial^{+}} \psi_{+}(x) .
$$

Note that $\tilde{Q}_{5}^{L}=Q_{5}^{L}$, these results are consistent with the definition of the light-front chiral transformation, eqs. (2.8 - 2.9). It shows that the usual chiral transformation eq. (2.7) (which corresponds to $\left[\psi(x), Q_{5}\right]=\gamma_{5} \psi(x)$ ) is invalid for light-front theory. 


\section{Chiral anomalies}

Before we discuss the physical implication of the new obtained light-front axial-vector current for QCD, we should derive in this section the anomaly associated with this lightfront axial-vector current. In equal-time frame, the chiral anomaly is originated from vacuum effect. In the light-front theory, the vacuum is occupied only by $k^{+}=0$ particles. With the help of infrared regularization of longitudinal light-front momentum, the lightfront vacuum is ensure to be trivial as the bare vacuum [1, 2]. Now we use the different vacuum structure and a new light-front axial-vector current to derive the chiral anomaly. There are three methods to derive the chiral anomaly of axial-vector current, namely the point-splitting technique [10, 11, 12], the triangle diagrams 112, 13, 14, 15] and the path-integral method [16]. The point-splitting technique has been used to calculate chiral anomaly in two and four dimensional light-front QED, where the conventional axial-vector current is used [17]. In our work, we shall use the point-splitting technique to calculate chiral anomaly of the associated new light-front axial-vector current in light-front QCD.

The light-front axial-vector current is also a composite operator built out of quark fields. The products of local operators are often singular. To regularize the axial-vector current, we introduce a small separation of the two quarks and insert the Wilson line to keep the operator being locally gauge invariant. At the end of calculation, we then carefully take $\epsilon$ to zero. It must be pointed out that after we choose the gauge $A_{a}^{+}=0$, the gauge degrees of freedom are fixed except for possible residual gauge transformations. The requirement of gauge invariance means that of the residual gauge transformation,

$$
\begin{aligned}
& \psi^{\prime}(x)=\mathrm{e}^{i \alpha(x) T^{a}} \psi(x), \\
& A_{a}^{\prime \mu}(x)=A_{a}^{\mu}(x)+\frac{1}{g} \partial^{\mu} \alpha_{a}(x)+f^{a b c} A_{b}^{\mu}(x) \alpha_{c}(x),
\end{aligned}
$$

where $\alpha_{a}(x)$ is the gauge function that obeys $\partial^{+} \alpha_{a}(x)=0$ such that the light-front gauge $A_{a}^{+}=0$ is guaranteed. Thus, from eq. (2.19), the gauge invariant light-front axial-vector current is given by

$$
\begin{aligned}
\tilde{j}_{5}^{\mu}= & \lim _{\epsilon \rightarrow 0}\left\{\bar{\psi}(x+\epsilon / 2) \gamma^{\mu} \gamma_{5} \mathcal{W}(A) \psi(x-\epsilon / 2)\right. \\
& \left.-m \bar{\psi}(x+\epsilon / 2) \gamma^{\mu} \gamma_{5} \gamma^{+} \mathcal{W}(A) \frac{1}{i \partial^{+}} \psi(x-\epsilon / 2)\right\},
\end{aligned}
$$

where $\mathcal{W}(A)=\exp \left[i g \int_{x-\epsilon / 2}^{x+\epsilon / 2} \mathrm{~d} z \cdot A(z)\right]$ is the Wilson line. We write down the each component of above current in terms of $\psi_{+}$and $\psi_{-}$

$$
\begin{aligned}
\tilde{j}_{5}^{+}= & 2 \lim _{\epsilon \rightarrow 0}\left\{\psi_{+}^{\dagger}\left(y_{+}\right) \gamma_{5} \mathcal{W}(A) \psi_{+}\left(y_{-}\right)\right\} \\
\tilde{j}_{5}^{-}= & 2 \lim _{\epsilon \rightarrow 0}\left\{\psi_{-}^{\dagger}\left(y_{+}\right) \gamma_{5} \mathcal{W}(A) \psi_{-}\left(y_{-}\right)-4 m \psi_{-}^{\dagger}\left(y_{+}\right) \gamma_{5} \gamma^{0} \mathcal{W}(A) \frac{1}{i \partial^{+}} \psi_{+}\left(y_{-}\right)\right\} \\
\tilde{j}_{5}{ }^{i}= & \lim _{\epsilon \rightarrow 0}\left\{\psi_{+}^{\dagger}\left(y_{+}\right) \gamma^{0} \gamma^{i} \gamma_{5} \mathcal{W}(A) \psi_{-}\left(y_{-}\right)+\psi_{-}^{\dagger}\left(y_{+}\right) \gamma^{0} \gamma^{i} \gamma_{5} \mathcal{W}(A) \psi_{+}\left(y_{-}\right)\right. \\
& \left.-2 m \psi_{+}^{\dagger}\left(y_{+}\right) \gamma^{i} \gamma_{5} \mathcal{W}(A) \frac{1}{i \partial^{+}} \psi_{+}\left(y_{-}\right)\right\}
\end{aligned}
$$


where $y_{ \pm}=x \pm \epsilon / 2$ and $\psi_{-}$is given by eq. (2.16). Then we calculate directly the divergence of the above light-front axial-vector current, $\partial_{\mu} \tilde{j}_{5}^{\mu}=\frac{1}{2} \partial^{-} \tilde{j}_{5}^{+}+\frac{1}{2} \partial^{+} \tilde{j}_{5}^{-}-\partial^{i} \tilde{j}_{5}^{i}$. Note that these derivative terms, $\partial^{-} \psi_{+}, \partial^{-} \psi_{-}$and their complex conjugate, can be substituted by using the light-front fermion constraint eq. (2.16) and the equation of motion

$$
\left(i \partial^{-}+g A^{-}\right) \psi_{+}=\left(i \vec{\alpha}_{\perp}+g \vec{\alpha}_{\perp} \cdot \vec{A}_{\perp}+\beta m\right) \psi_{-},
$$

where $A^{-}$satisfies light-front gluon constraint:

$$
A_{a}^{-}=2 \frac{\partial^{i}}{\partial^{+}} A_{a}^{i}-2 g\left(\frac{1}{\partial^{+}}\right)^{2}\left(f^{a b c} A_{b}^{i} \partial^{+} A_{c}^{i}+2 \psi_{+}^{\dagger} T^{a} \psi_{+}\right) .
$$

A tedious but straightforward calculation shows that

$$
\begin{aligned}
\partial_{\mu} \tilde{j}_{5}^{\mu} & =i g \psi_{+}^{\dagger}\left(y_{+}\right)\left\{\mathcal{W}(A) A^{-}\left(y_{-}\right)-A^{-}\left(y_{+}\right) \mathcal{W}(A)+\epsilon^{\nu} \partial^{-} A_{\nu}(x) \mathcal{W}(A)\right\} \gamma_{5} \psi_{+}\left(y_{-}\right) \\
& +i g \psi_{+}^{\dagger}\left(y_{+}\right)\left\{\mathcal{W}(A) A^{i}\left(y_{-}\right)-A^{i}\left(y_{+}\right) \mathcal{W}(A)+\epsilon^{\nu} \partial^{i} A_{\nu}(x) \mathcal{W}(A)\right\} \gamma^{i} \gamma^{0} \gamma_{5} \psi_{-}\left(y_{-}\right) \\
& +i g \psi_{-}^{\dagger}\left(y_{+}\right)\left\{\mathcal{W}(A) A^{i}\left(y_{-}\right)-A^{i}\left(y_{+}\right) \mathcal{W}(A)+\epsilon^{\nu} \partial^{i} A_{\nu}(x) \mathcal{W}(A)\right\} \gamma^{i} \gamma^{0} \gamma_{5} \psi_{+}\left(y_{-}\right) \\
& +i g \psi_{-}^{\dagger}\left(y_{+}\right)\left\{\epsilon^{\nu} \partial^{+} A_{\nu}\left(y_{+}\right) \mathcal{W}(A)\right\} \gamma_{5} \psi_{-}\left(y_{-}\right) \\
& +2 m g \psi_{+}^{\dagger}\left(y_{+}\right) \gamma^{i} \gamma_{5} \mathcal{W}(A)\left(\frac{1}{\partial^{+}} A^{i}\left(y_{-}\right)\right) \psi_{+}\left(y_{-}\right) \\
& +2 m g \psi_{+}^{\dagger}\left(y_{+}\right)\left(\mathcal{W}(A) A^{i}\left(y_{-}\right)-A^{i}\left(y_{+}\right) \mathcal{W}(A)\right) \gamma^{i} \gamma_{5} \frac{1}{\partial^{+}} \psi_{+}\left(y_{-}\right) \\
& +2 m g \psi_{-}^{\dagger}\left(y_{+}\right) \gamma^{0} \gamma_{5}\left(\epsilon^{\nu} \partial^{+} A_{\nu}(x)\right) \mathcal{W}(A) \frac{1}{\partial^{+}} \psi_{+}\left(y_{-}\right) \\
& +2 m g \psi_{+}^{\dagger}\left(y_{+}\right) \gamma^{i} \gamma_{5}\left(\epsilon^{\nu} \partial^{i} A_{\nu}(x)\right) \mathcal{W}(A) \frac{1}{\partial^{+}} \psi_{+}\left(y_{-}\right)
\end{aligned}
$$

Here $\epsilon^{\mu}$ is small, we take an expansion of the independent gauge fields $A^{i}$ with respect to $\epsilon$,

$$
A^{i}\left(x \pm \frac{\epsilon}{2}\right) \approx A^{i}(x) \pm \frac{\epsilon^{\beta}}{2} \partial_{\beta} A^{i}(x)
$$

The expansion of the dependent component of the gauge fields $A_{a}^{-}$can be determined from the light-front gluon constraint eq. (3.4):

$$
\begin{aligned}
A_{a}^{-}\left(x \pm \frac{\epsilon}{2}\right)= & 2 \frac{\partial^{i}}{\partial^{+}} A_{a}^{i}\left(x \pm \frac{\epsilon}{2}\right) \\
& -2 g\left(\frac{1}{\partial^{+}}\right)^{2}\left[f^{a b c} A_{b}^{i}\left(x \pm \frac{\epsilon}{2}\right) \partial^{+} A_{c}^{i}\left(x \pm \frac{\epsilon}{2}\right)+2 \psi_{+}^{\dagger}\left(x \pm \frac{\epsilon}{2}\right) T^{a} \psi_{+}\left(x \pm \frac{\epsilon}{2}\right)\right] \\
\approx & 2 \frac{\partial^{i}}{\partial^{+}} A_{a}^{i}(x)-2 g\left(\frac{1}{\partial^{+}}\right)^{2}\left[f^{a b c} A_{b}^{i}(x) \partial^{+} A_{c}^{i}(x)+2 \psi_{+}^{\dagger}(x) T^{a} \psi_{+}(x)\right] \\
& \pm \frac{\epsilon^{\beta}}{2} \partial_{\beta}\left\{2 \frac{\partial^{i}}{\partial^{+}} A_{a}^{i}(x)-2 g\left(\frac{1}{\partial^{+}}\right)^{2}\left[f^{a b c} A_{b}^{i}(x) \partial^{+} A_{c}^{i}(x)+2 \psi_{+}^{\dagger}(x) T^{a} \psi_{+}(x)\right]\right\} \\
= & A_{a}^{-}(x) \pm \frac{\epsilon^{\beta}}{2} \partial_{\beta} A_{a}^{-}(x) .
\end{aligned}
$$


Thus, keeping in mind the expansion is taken up to the order $\epsilon$ :

$$
\begin{aligned}
& A_{\alpha}\left(x \pm \frac{\epsilon}{2}\right) \approx A_{\alpha}(x) \pm \frac{\epsilon^{\beta}}{2} \partial_{\beta} A_{\alpha}, \\
& \mathcal{W}(A) \approx 1+i g \epsilon^{\alpha} A_{\alpha}(x),
\end{aligned}
$$

we can reduce eq. (3.5) to

$$
\begin{aligned}
\partial_{\mu} \tilde{j}_{5}^{\mu}= & \lim _{\epsilon \rightarrow 0}\left\{i g \epsilon^{\nu} F_{a \mu \nu} \bar{\psi}\left(x+\frac{\epsilon}{2}\right) \gamma^{\mu} \gamma_{5} T^{a} \psi\left(x-\frac{\epsilon}{2}\right)\right. \\
& -m g \epsilon^{\nu} F_{a}{ }^{i}{ }_{\nu} \bar{\psi}\left(x+\frac{\epsilon}{2}\right) \gamma^{+} \gamma^{i} \gamma_{5} T^{a} \psi\left(x-\frac{\epsilon}{2}\right) \\
& \left.-m g \epsilon^{\nu} F_{a}{ }^{+}{ }_{\nu} \bar{\psi}\left(x+\frac{\epsilon}{2}\right) \gamma^{-} \gamma_{5} \gamma^{0} T^{a} \frac{1}{\partial^{+}} \psi\left(x-\frac{\epsilon}{2}\right)\right\} \\
& +\lim _{\epsilon \rightarrow 0}\left\{2 m g \psi_{+}^{\dagger}\left(x+\frac{\epsilon}{2}\right) \gamma^{i} \gamma_{5} \mathcal{W}(A)\left(\frac{1}{\partial^{+}} A^{i}\left(x-\frac{\epsilon}{2}\right)\right) \psi_{+}(x-\epsilon / 2)\right\},
\end{aligned}
$$

where $T^{a}$ is a color $\mathrm{SU}(3)$ generator and $F_{\mu \nu}=\partial_{\mu} A_{\nu}-\partial_{\nu} A_{\mu}-i g\left[A_{\mu}, A_{\nu}\right]$. The last term in eq. (3.9) is regular as well as $\epsilon$ approaching to zero, which gives us the divergence in eq. (2.20). Consider the vacuum expectation value of $\partial_{\mu} \tilde{j}_{5}^{\mu}$. The first term of eq. (3.9) is

$$
\begin{aligned}
& i g \epsilon^{\nu} F_{a \mu \nu}\left\langle 0\left|\bar{\psi}\left(x+\frac{\epsilon}{2}\right) \gamma^{\mu} \gamma_{5} T^{a} \psi\left(x-\frac{\epsilon}{2}\right)\right| 0\right\rangle \\
& =i g \epsilon^{\nu} F_{a \mu \nu} \operatorname{Tr}\left\{T^{a} \operatorname{Tr}\left\{\gamma^{\mu} \gamma_{5} \tilde{G}\left(x-\frac{\epsilon}{2}, x+\frac{\epsilon}{2}\right)\right\}\right\} .
\end{aligned}
$$

With the existence of background field, the propagator of fermions can be expanded as

$$
\begin{aligned}
\tilde{G}\left(x-\frac{\epsilon}{2}, x+\frac{\epsilon}{2}\right) & =\left\langle 0\left|\psi\left(x-\frac{\epsilon}{2}\right) \bar{\psi}\left(x+\frac{\epsilon}{2}\right)\right| 0\right\rangle \\
& =\tilde{S}(-\epsilon)+\int \mathrm{d}^{4} y \tilde{S}\left(x-\frac{\epsilon}{2}-y\right)(i g \not A(y)) \tilde{S}\left(y-x-\frac{\epsilon}{2}\right),
\end{aligned}
$$

where

$$
\tilde{S}(x)=\int \frac{\mathrm{d}^{4} p}{(2 \pi)^{4}} \mathrm{e}^{-i p \cdot x}\left[\frac{i(\not p+m)}{p^{2}-m^{2}+i \varepsilon}-\frac{1}{2} i \frac{\gamma^{+}}{p^{+}}\right]
$$

is the light-front propagator of quark field [18]. Thus, eq. (3.10) becomes

$$
\begin{aligned}
& \left\langle 0\left|\psi\left(x+\frac{\epsilon}{2}\right) \gamma^{\mu} \gamma_{5} T^{a} \psi\left(x-\frac{\epsilon}{2}\right)\right| 0\right\rangle \\
& =-\operatorname{Tr}\left\{T^{a} T^{b}\right\} \int \frac{\mathrm{d}^{4} p}{(2 \pi)^{4}} \int \frac{\mathrm{d}^{4} k}{(2 \pi)^{4}} e^{-i k \cdot \epsilon} e^{-i p \cdot(x+\epsilon / 2)} \operatorname{Tr}\left\{\gamma^{\mu} \gamma_{5} \tilde{S}(p+k)\left(i g \mathcal{A}^{b}(p)\right) \tilde{S}(k)\right\} \\
& =i g \operatorname{Tr}\left\{T^{a} T^{b}\right\} \int \frac{\mathrm{d}^{4} p}{(2 \pi)^{4}} \int \frac{\mathrm{d}^{4} k}{(2 \pi)^{4}} e^{-i k \cdot \epsilon} e^{-i p \cdot(x+\epsilon / 2)} \\
& \left\{4 i \epsilon^{\mu \alpha \beta \rho} \frac{(p+k)_{\alpha} A_{\beta}^{b}(p) k_{\rho}}{\left[(p+k)^{2}-m^{2}+i \varepsilon\right]\left[k^{2}-m^{2}+i \varepsilon\right]}-2 i \epsilon^{\mu \alpha \beta+} \frac{(p+k)_{\alpha} A_{\beta}^{b}(p)}{\left[(p+k)^{2}-m^{2}+i \varepsilon\right] k^{+}}\right. \\
& \left.+2 i \epsilon^{\mu \alpha \beta+} \frac{A_{\beta}^{b}(p) k_{\alpha}}{\left[k^{2}-m^{2}+i \varepsilon\right] k^{+}}\right\} .
\end{aligned}
$$

As $\epsilon \rightarrow 0$, the three terms in eq. (3.13) are proportional to $k, k^{2}$ and $k^{2}$ respectively, in the integration of $\mathrm{k}$. Thus, the main contribution comes from the large $k$ region, namely 
$p<<k$. With such a consideration [19], the last two terms of eq. (3.13) cancel each other and the first term is reduced to

$$
\int \frac{\mathrm{d}^{4} p}{(2 \pi)^{4}} e^{-i p \cdot x} p_{\alpha} A_{\beta}^{b}(p) \int \frac{\mathrm{d}^{4} k}{(2 \pi)^{4}} e^{-i k \cdot \epsilon} \frac{k_{\rho}}{k^{4}}=\partial_{\alpha} A_{\beta}^{a}(x)\left(\frac{-i}{8 \pi^{2}} \frac{\epsilon_{\rho}}{\epsilon^{2}}\right) .
$$

One can easily verify that the above result is singular as $\epsilon \rightarrow 0$, as we mention in the front of this section. But the combination with the term $\epsilon^{\nu}$ in eq. (3.10) gives a finite contribution:

$$
\lim _{\epsilon \rightarrow 0} \frac{\epsilon^{\nu} \epsilon_{\rho}}{\epsilon^{2}}=\frac{1}{4} g_{\rho}^{\nu}
$$

Substituting these results into eq. (3.13), we have

$$
i g \epsilon^{\nu} F_{a \mu \nu}\left\langle 0\left|\psi\left(x+\frac{\epsilon}{2}\right) \gamma^{\mu} \gamma_{5} T^{a} \psi\left(x-\frac{\epsilon}{2}\right)\right| 0\right\rangle=-\frac{g^{2}}{16 \pi^{2}} \epsilon^{\alpha \beta \mu \nu} \operatorname{Tr}\left\{F_{\alpha \beta} F_{\mu \nu}\right\} .
$$

A similar calculation shows that the other two terms in eq. (3.9) give no contribution. Thus, the anomalous term for the light-front axial-vector current is identical to that in equal-time formalism. Then the divergence of light-front axial-vector current can be written as follows:

$$
\partial_{\mu} \tilde{j}_{5}^{\mu}=m g \bar{\psi} \gamma_{5} \gamma^{+}\left(\frac{1}{\partial^{+}} \vec{\gamma}_{\perp} \cdot \vec{A}_{\perp}\right) \psi-\frac{g^{2}}{16 \pi^{2}} \epsilon^{\alpha \beta \mu \nu} \operatorname{Tr}\left\{F_{\alpha \beta} F_{\mu \nu}\right\} .
$$

Using eq. 2.11) and eq. (3.17) can be reduced to the conventional form:

$$
\partial_{\mu} j_{5}^{\mu}=2 i m \bar{\psi} \gamma_{5} \psi-\frac{g^{2}}{16 \pi^{2}} \epsilon^{\alpha \beta \mu \nu} \operatorname{Tr}\left\{F_{\alpha \beta} F_{\mu \nu}\right\}
$$

namely, eq. (3.17) and eq. (3.18) are again consistent each other even if the chiral anomaly is included.

\section{Physical implication of light-front axial-vector current}

Now we shall discuss the physical implication of the new light-front axial-vector current. In this section, we study physical implication in light-front QCD from two aspects, the spontaneous symmetry breaking (SSB) and the hadronic transition matrix element of axialvector current. In equal-time formulation, the divergence of axial-vector current eq. (1.1) is considered as an interpolating pion field through the partially conserved axial-vector current (PCAC) hypothesis:

$$
\partial_{\mu} j_{5}^{\mu}(x)=m_{\pi}^{2} f_{\pi} \phi(x)
$$

where $\phi(x)$ is the pion field operator, $f_{\pi}$ the pion decay constant and $m_{\pi}$ the pion mass. Comparing eq. (1.1) with eq. (4.1), one can see that the composite operator of quark fields $\bar{\psi} i \gamma_{5} \psi$ is the interpolating pion field, and the limit of quark mass $m \rightarrow 0$ implies $m_{\pi}=0$ i.e., $\pi$ is massless in the massless quark theory, as a result of the spontaneous chiral symmetry breaking. This is also manifested from the commutation of the static 
chiral charge $Q_{5}$ [defined in equal-time formalism $Q_{5}=\int \mathrm{d}^{3} x j_{5}^{0}(x)$ ] with the interpolating pion field operator:

$$
\left[Q_{5}, \bar{\psi}(x) i \gamma_{5} \psi(x)\right]_{x^{0}=0}=-2 i \bar{\psi}(x) \psi(x) .
$$

The nonzero value of the vacuum expectation $\langle\bar{\psi} \psi\rangle$ (chiral condensate) is the character of spontaneous chiral symmetry breaking $\left(Q_{5}|\Omega\rangle=|\Phi\rangle\right)$, namely the chiral charge creates massless Goldstone bosons from the vacuum as a consequence of spontaneous chiral symmetry breaking. These discussion seems to be at variance with the light-front framework because of $\tilde{Q}_{5}^{L}|0\rangle=0$. To be more explicit, we check the commutation of the light-front chiral charge $\left(\tilde{Q}_{5}^{L}\right)$ with the interpolating pion field $\left(\bar{\psi} i \gamma_{5} \psi\right)$. The result is

$$
\begin{aligned}
{\left[\tilde{Q}_{5}^{L}, \bar{\psi}(x) i \gamma_{5} \psi(x)\right]_{x^{+}=0}=} & -2 i \bar{\psi}(x) \psi(x) \\
& +m\left[\bar{\psi}(x) \gamma^{+}\left(\frac{1}{\partial^{+}} \psi(x)\right)-\left(\frac{1}{\partial^{+}} \bar{\psi}(x)\right) \gamma^{+} \psi(x)\right] .
\end{aligned}
$$

Comparing with eq. (4.2), there is an extra term $m\left[\bar{\psi}(x) \gamma^{+}\left(\frac{1}{\partial^{+}} \psi(x)\right)-\left(\frac{1}{\partial^{+}} \bar{\psi}(x)\right) \gamma^{+} \psi(x)\right]$. The vacuum expectation value of the left-hand side of eq. (4.3) must vanish due to the fact that the light-front chiral charge annihilates the vacuum, $\tilde{Q}_{5}^{L}|0\rangle=0$. Thus the right-hand side must vanishes as well. This leads to

$$
\langle 0|\bar{\psi} \psi| 0\rangle=\frac{-i m}{2}\left\langle 0\left|\left[\bar{\psi} \gamma^{+}\left(\frac{1}{\partial^{+}} \psi\right)-\left(\frac{1}{\partial^{+}} \bar{\psi}\right) \gamma^{+} \psi\right]\right| 0\right\rangle,
$$

namely the quark condensate in light-front theory can be non-zero even if the chiral charge annihilates the vacuum. This is no surprise because the right-hand side of eq. (4.3) contains $\left(1 / \partial^{+}\right)$and therefore is infrared singular. The renormalization of the infrared divergence (related to the light-front zero mode in QCD) can give $\langle 0|\bar{\psi} \psi| 0\rangle$ a nonzero value even in the chiral limit $m \rightarrow 0$. However, for Abelian QED, since $\psi$ (electron) is a physical particle field, it should vanish in spacial infinity. Thus, eq. (2.3) for $1 / \partial^{+}$which leads to an antisymmetric boundary condition for $\psi$ at the longitudinal infinity cannot apply to QED, and as a result, the light-front longitudinal infrared problem in QED is trivial (within the perturbation theory) and cannot generate a non-zero chiral condensate of eq. (4.4).

This result is analogous to Higgs mechanism of SSB in the light-front formulation of the standard model [20] that moving the complicity of the vacuum state into the operator level. However, the straightforward Higgs mechanism of SSB by a simple shift of the scalar field cannot be applied to QCD. Here, such a realization is naturally carried out from the new light-front axial-vector current derived from the consistent light-front chiral transformation. While the possible non-vanishing quark condensate is associated with the infrared divergence in light-front theory, namely the renormalization treatment of the light-front zero mode in QCD. Unfortunately, a practical scheme of the light-front infrared renormalization for QCD still remains to be solved [3].

On the other hand, an arbitrary hadronic transition matrix element of axial-vector current can be separated into a pole term plus a non-pole term:

$$
\left\langle B\left|j_{5}^{\mu}(0)\right| A\right\rangle=\frac{i f_{\pi} q^{\mu}}{q^{2}-m_{\pi}^{2}}\langle B, \pi \mid A\rangle+\left\langle B\left|j_{5}^{\mu}\right| A\right\rangle_{N}
$$


where $p_{A(B)}$ is the momentum carried by hadron $\mathrm{A}(\mathrm{B})$ and $q=p_{A}-p_{B}$. This indicates that the axial-vector current $j_{5}^{\mu}$ contains two resources, one describes the pion mode in vacuum, and the other describes hadronic transitions involving pion. As it has been discussed in [6], using the PCAC hypothesis, eq. (4.1), one can show from eq. (4.5) that

$$
\langle B \pi \mid A\rangle=i q_{\mu}\left\langle B\left|j_{5}^{\mu}\right| A\right\rangle_{N} / f_{\pi} .
$$

Substituting eq. (4.6) into eq. (4.5), we obtain

$$
\left\langle B\left|j_{5}^{\mu}(0)\right| A\right\rangle=\left(g_{\nu}^{\mu}-\frac{q^{\mu} q_{\nu}}{q^{2}-m_{\pi}^{2}}\right)\left\langle B\left|j_{5}^{\nu}\right| A\right\rangle_{N}
$$

If we take the limit, $q^{+}, \vec{q}_{\perp} \rightarrow 0$, then

$$
\left\langle B\left|Q_{5}^{L}\right| A\right\rangle=\left.\frac{\left\langle p_{B}|| p_{A}\right\rangle}{p_{A}^{+}+p_{B}^{+}}\left\langle B\left|j_{5}^{+}\right| A\right\rangle_{N}\right|_{q^{+}=\vec{q}_{\perp}=0},
$$

where $\left\langle p_{B}|| p_{A}\right\rangle=2(2 \pi)^{3} p_{A}^{+} \delta\left(p_{A}^{+}-p_{B}^{+}\right) \delta^{2}\left(\vec{p}_{A \perp}-\vec{p}_{B \perp}\right)$. The eq. (4.8) implies that hadronic transition matrix elements of light-front chiral charge is only contributed by the non-pole term. In other words, the light-front chiral charge does not contain the pole contribution.

This property has also recently been discussed phenomenologically in the light-front formalism, namely, the axial current $j_{5}^{\mu}$ contains an interpolating field of the $\pi$-field plus a non-pole term: $j_{5}^{\mu}=-f_{\pi} \partial_{\mu} \pi+\tilde{j}_{5}^{\mu}$ and the light-front chiral charge $Q_{5}^{L}=\frac{1}{2} \int d x^{-} d^{2} x_{\perp} \bar{j}_{5}^{+}$is constructed only from the non-pole term [21]. Now, we shall show that the light-front axialvector current $\tilde{j}_{5}^{\mu}$ derived in this paper just corresponds to the non-pole contribution and the interpolating $\pi$-field $\left(\partial^{\mu} \pi\right)$ is related to $\hat{j}_{5}^{\mu}=j_{5}^{\mu}-\tilde{j}_{5}^{\mu}=m \bar{\psi} \gamma^{\mu} \gamma_{5} \gamma^{+} \frac{1}{i \partial^{+}} \psi$ in eq. (2.19).

Existence of the pole term means that the chiral charge can create a pion from vacuum or annihilate a pion [see eq. (4.2)]. To extract the pole contribution from the axial-vector current in light-front theory, we consider the following matrix element:

$$
\left\langle 0\left|j_{5}^{\mu}\right| \pi(p)\right\rangle=\left\langle 0\left|\tilde{j}_{5}^{\mu}\right| \pi(p)\right\rangle+\left\langle 0\left|\hat{j}_{5}^{\mu}\right| \pi(p)\right\rangle,
$$

here we have used the relation between the conventional and the light-front axial-vector currents that has been shown in eq. (2.19). From eq. (4.5), the hadronic matrix element of the conventional axial-vector current contains both the pole term and the non-pole term. Thus, for the matrix element $\left\langle 0\left|j_{5}^{\mu}\right| \pi(p)\right\rangle$ in eq. (4.9) only contains the pole contribution. Meanwhile, the right-hand side of the above equation is naturally separated into two terms based on eq. (2.19). An explicit calculation shows that the divergence of $\hat{j}_{5}^{\mu}$ :

$$
\begin{aligned}
\left\langle 0\left|\partial_{\mu} \hat{j}_{5}^{\mu}\right| \pi\right\rangle= & -m g\left\langle 0\left|\bar{\psi} \gamma_{5} \gamma^{+}\left(\frac{1}{\partial^{+}} \vec{\gamma}_{\perp} \cdot \vec{A}_{\perp}\right) \psi\right| \pi\right\rangle \\
& +2 m\left\langle 0\left|\bar{\psi} i \gamma_{5} \psi\right| \pi\right\rangle .
\end{aligned}
$$

The first term cancels with $\left\langle 0\left|\partial_{\mu} \tilde{j}_{5}^{\mu}\right| \pi\right\rangle$ [see eq. (2.20)] and the second term corresponds to the divergence of the conventional axial-vector current. Using the PCAC hypothesis, we have

$$
\begin{aligned}
-i p_{\mu}\left\langle 0\left|\hat{j}_{5}^{\mu}(x)\right| \pi(p)\right\rangle & =\left\langle 0\left|\partial_{\mu} j_{5}^{\mu}(x)\right| \pi(p)\right\rangle \\
& =m_{\pi}^{2} f_{\pi}\langle 0|\phi(x)| \pi(p)\rangle .
\end{aligned}
$$


Equivalently, we can write the above equation as

$$
\left\langle 0\left|\hat{j}_{5}^{\mu}(x)\right| \pi(p)\right\rangle=-f_{\pi}\left\langle 0\left|\partial^{\mu} \phi(x)\right| \pi(p)\right\rangle .
$$

This means that the term $\hat{j}_{5}^{\mu}$ in eq. (2.19) is related to the interpolating pion field operator, and the hadronic transition matrix element of the light-front axial-vector current $\tilde{j}_{5}^{\mu}$ is only related to the non-pole contribution. This also explain why the light-front chiral charge annihilate the vacuum.

\section{Summary}

In summary, the chiral transformation defined in light-front theory is very different from that in equal-time frame. The light-front fermion field is divided into up and down components, only the up component is the dynamical variable and the down component is a constraint. Hence, the proper chiral transformation on light-front is defined by eq. (2.8). In the free fermion theory, the light-front axial-vector current is conserved, no matter it is massless or massive.

As far we have learned from current algebra is the properties of current algebra from free quark theory. In the early development of QCD, it was argued that the properties remain true when the interaction between quark and gluon is switched on because the divergence depends only on quark mass. This seems to contradict with light-front currents which are known to be dynamical dependent. We have derived the divergence equation of axial-vector current in light-front QCD. The light-front axial-vector current $\tilde{j}_{5}^{\mu}$ is the same as the free fermion theory except that the light-front fermion constraint is given by eq. (2.16). The result presented in this paper shows that the chiral symmetry is broken by the mixing of quark mass and quark-gluon interaction i.e. the helicity flip interaction. We also study the quantum effect of the new axial-vector current associated with the chiral transformation in light-front theory. We show that the anomaly in light-front theory is consistent with the case in equal-time frame.

Furthermore, we show in this formulism that the light-front chiral charge annihilates the vacuum but the chiral condensate can be nonzero. Meanwhile, the light-front axialvector current $\tilde{j}_{5}^{\mu}$ can be obtained from the axial-vector current $j_{5}^{\mu}$ by subtracting an additional term $\hat{j}_{5}^{\mu}$. The term $\hat{j}_{5}^{\mu}$ corresponds to the pole contribution which is very complicated in QCD, while, the light-front axial-vector current corresponds to the non-pole contribution of the conventional axial-vector current that smoothly describes various pion transitions even in the chiral limit. As a conclusion, we believe that the chiral dynamics in light-front QCD can largely simplify hadronic physics.

\section{Acknowledgments}

This work was supported in part by the National Science Council of ROC under Contract Nos. NSC-92-2112-M-006-024 and NSC-92-2112-M-001-030. WMZ would also like to thank A. Harindranath for the fruitful discussion in the beginning of this work. 


\section{References}

[1] K. G. Wilson, T. S. Walhout, A. Harindranth, W. M. Zhang, R. J. Perry and S. D. Glazek, Phys. Rev. D 49 (1994) 6720.

[2] W. M. Zhang and A. Harindranath, Phys. Rev. D 48 (1993) 4868; ibid. 48 (1993) 4881.

[3] K. G. Wilson and M. Brisudova, hep-th/9411008.

[4] M. Burkardt and H. El-Khozondar, Phys. Rev. D 55 (1997) 6514.

[5] M. Burkardt, Phys. Rev. D 58 (1998) 096015.

[6] R. Carlitz, D. Heckathorn, J. Kaur and W.-K. Tung, Phys. Rev. D 11 (1975) 1234.

[7] D. Mustaki, hep-ph/9404206.

[8] K. Itakura and S. Maedan, Phys. Rev. D 61 (2000) 045009; ibid. 62 (2000) 105016; Prog. Theor. Phys. 105 (2001) 537.

[9] L. Martinovič and J. P. Vary, Phys. Rev. D 64 (2001) 105016.

[10] C. R. Hagen, Phys. Rev. 177 (1969) 2622.

[11] R. Jackiw and K. Johnson, Phys. Rev. 182 (1969) 1459.

[12] S. B. Treiman, R. Jackiw, B. Zumino and E. Witten, Current Algebra and Anomalies (World Scientific, Singapore, 1985).

[13] S. L. Adler, Phys. Rev. 177 (1969) 2426.

[14] S. L. Adler and W. A. Bardeen, Phys. Rev. 182 (1969) 1517.

[15] S. L. Adler and D. G. Boulware, Phys. Rev. 184 (1969) 1740.

[16] K. Fujikawa, Phys. Rev. Lett. 42 (1979) 1195; Phys. Rev. D 21 (1980) 2848.

[17] L. Martinovič and J. P. Vary, Nucl. Phys. 90 (Proc. Suppl.) (2000) 57.

[18] S. J. Chang and T. M. Yan, Phys. Rev. D 7 (1973) 1147.

[19] M. E. Peskin and D. V. Schroeder, An Introduction to Quantum Field Theory, (Addison-Wesley, 1995).

[20] P. P. Srivastava and S. J. Brodsky, Phys. Rev. D 66 (2002) 045019.

[21] Y. Kim, S. Tsujimaru and K. Yamawaki, Phys. Rev. Lett. 74 (1995) 4771; S. Tsujimaru and K. Yamawaki, Phys. Rev. D 57 (1998) 4942. 